\title{
Nonlinear Uncertain HIV-1 Model Controller by Using Control Lyapunov Function
}

\author{
Fatma A. Alazabi, Mohamed A. Zohdy \\ Department of Electrical and Computer Engineering, Oakland University, Rochester, USA \\ Email: faalazab@oakland.edu
}

Received May 4, 2012; revised May 15 2012; accepted May 25, 2012

\begin{abstract}
In this paper, we introduce a new Control Lyapunov Function (CLF) approach for controlling the behavior of nonlinear uncertain HIV-1 models. The uncertainty is in decay parameters and also external control setting. CLF is then applied to different strategies. One such strategy considers input into infected cells population stage and the other considers input into a virus population stage. Furthermore, by adding noise to the HIV-1 model a realistic comparison between control strategies is presented to evaluate the system's dynamics. It has been demonstrated that nonlinear control has effectiveness and robustness, in reducing virus loading to an undetectable level.
\end{abstract}

Keywords: HIV-1 Infection Model; Control Lyapunov Function (CLF); Control Strategy; Uncertain Parameters; Noise Effect

\section{Introduction}

Modeling physical or biological phenomena for any dynamic system needs to take into account the nature of connection between the parameters of the dynamic system and the observed solution [1]. The function of these parameters is reflecting the characteristics of studied phenomena such that death rate of productive infected $\mathrm{CD}^{+} \mathrm{T}$ cells for the HIV model. Therefore, it is valuable to know about how perturbations in these parameters present themselves in the solution. There are many papers for HIV modeling that consider the importance of parameters into a system's dynamic [2-4]. For example, in [5], a new mathematical model is presented to analyze many details on HIV-1 viral load data collected from five infected patients that were administrated using protease inhibitor. Based on data provided in [5], many viral dynamics that can not only give the kinetics dynamic of HIV-1 disease but also give guidelines to develop new treatment strategy are investigated. Controlling HIV infection disease has been an interesting problem for many researchers [6-11]. It is well known [12,13] that a control Lyapunov function, if available, will be a convenient tool to analyze stability, evaluate the system's robustness to perturbations, or even to modify the design to enhance robustness or performance [14]. In this paper, a CLF approach for nonlinear uncertain HIV-1 model is introduced. The uncertainty is applied into system's decay parameters and external control. Also, two different strategies based on CLF are investigated. It has been shown that the first strategy is effective and has an ability to reduce virus concentration to an undetectable level even under uncertainty and noise effect.

\section{Theory of Control Lyapunov Function (CLF)}

A function $V(x)$ is said to be a Lyapunov function for a given system of vector state equations:

$$
\dot{x}=F(x) \text { with } F(0)=0, x \in R^{n}
$$

If it is class $C^{1}$ and there exists a neighborhood $Q$ of the origin such that [15]:

$$
\begin{aligned}
& V(0)=0 \text { and } V(x)>0 \text { for } x \in Q, \quad x \neq 0 \\
& \dot{V}(x)=\nabla V(x) F(x)<0 \text { for } x \in Q, \quad x \neq 0
\end{aligned}
$$

where: $\nabla V(x)=\left[\frac{\partial V(x)}{\partial x_{1}}, \frac{\partial V(x)}{\partial x_{2}}, \frac{\partial V(x)}{\partial x_{3}}, \ldots \ldots \frac{\partial V(x)}{\partial x_{n}}\right]$.

The classical Lyapunov stability theorem states that if Equation (1) has a suitable Lyapunov function, then the origin is globally asymptotically stable. Conversely, for any globally asymptotically stable system (1) with a continuous right hand side a Lyapunov function class $C^{\infty}$ can be constructed.

If we consider the control system:

$$
\dot{x}=f(x, u)
$$

$x \in \Omega \in R^{n}$ is the state vector. $u \in R^{m}$ is the control 
vector and is assumed continuously be stabilized. According to the above definition, a positive definite $C^{\infty}$ function exists such that:

$$
\inf (\nabla V(x) f(x, u))<0
$$

for each $x \neq 0$ in some neighborhood $Q$ of the origin.

As a result, if the function $V(x)$ of class $C^{\infty}$ satisfies Equations (2) and (5), then it's called a CLF [15].

\section{Selection of Suitable CLF}

It was shown in [16] that a first integral for the drift vector field, plus some controllability conditions can derive smooth asymptotically stabilizing control laws. This method has been introduced generally in $[15,17]$, and is usually called Jurdjevic-Quinn method [14]. The control strategy based on this requires selection of CLF such that $V(x)$ is semi-positive definite. Stability is guaranteed if the derivative of $V(x)$ is semi-negative definite. Considering the following system (linear in control and nonlinear in state):

$$
\dot{x}=f_{o}(x)+\sum_{i=1}^{m} u_{i} f_{i}(x)
$$

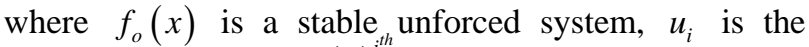
designated control, $f_{i}(x)^{i^{\text {th }}}$ is a smooth vector field in $R^{n}$.

We say that Equation (6) satisfies a Lyapunov condition of Jurdjevic-Quinn type if there are a neighborhood $Q$ of the origin and a $C^{\infty}$ function $V(x)$ such that [15]:

$$
\begin{aligned}
& V(x)>0 \text { for } x \in Q, \quad x \neq 0 \text { and } V(0)=0 \\
& \nabla V(x) f_{o}(x) \leq 0 \text { for } x \in Q
\end{aligned}
$$

Now, the derivative of $V(x)$ with respect to the closed loop system is given by [15]:

$$
\dot{V}(x)=\nabla V(x) f_{o}(x)-\sum_{i=1}^{m}\left[\nabla V(x) f_{i}(x)\right]^{2} \leq 0
$$

According to the Lyapunov control, a control function is selected as following:

$$
u_{i}(x)=-\nabla V(x) f_{i}(x), \quad i=1,2, \cdots, m
$$

\section{Basic HIV-1 Infection Model}

Parameters of HIV-1 infection models were estimated based on data provided by the Veterans Affairs hospital in West Haven, Connecticut, for a cohort of 338 people monitored for up to 2484 days [18]. This basic HIV-1 infection model is bilinear and has three states, namely uninfected cells $(x)$, infected cells $(y)$, and virus $(v)$ :

$$
\begin{aligned}
& \dot{x}=k_{1}-k_{2} x-k_{3} x v \\
& \dot{y}=k_{3} x v-k_{4} y \\
& \dot{v}=k_{5} y-k_{6} v
\end{aligned}
$$

Let $x=\left[x_{1}, x_{2}, x_{3}\right]^{T}=[x, y, v]^{T}$ and let $(\cdot)=\mathrm{d}() / \mathrm{d} t$, where $[\cdot]^{T}$ denotes transpose, Then:

$$
\begin{aligned}
& \dot{x}_{1}=f_{1}(\cdot)=k_{1}-k_{2} x_{1}-k_{3} x_{1} x_{3} \\
& \dot{x}_{2}=f_{2}(\cdot)=k_{3} x_{1} x_{3}-k_{4} x_{2} \\
& \dot{x}_{3}=f_{3}(\cdot)=k_{5} x_{2}-k_{6} x_{3}
\end{aligned}
$$

Figure 1 shows the dynamic response of HIV-1 infection model.

From Equation (12), $k_{1}$ is the supply rate of uninfected cells by the thymus, $k_{2}$ is the death rate of uninfected cells. $k_{3}$ is the rate of infection, $k_{4}$ is the death rate of infected cells, $k_{5}$ is the rate of virus production by infected cells, $k_{6}$ is the clearance rate of the virus. $x_{1}$ and $x_{2}$ are measured in (cells $/ \mathrm{mm}^{3}$ ) and $x_{3}$ is measured in (particles $/ \mathrm{mm}^{3}$ ). Also, from [18] we got Table 1.

\section{Robust CLF Controller Design}

Many control techniques have been applied for HIV treatment $[19,20]$, but here we are interested to develop a new control design based CLF. A stabilizing state feedback law can be found via a suitable semi-definite positive function $V(x)$ in two assumed cases as:

$$
\dot{x}=f_{o}(x)+\sum_{i=1}^{m} u_{i} f_{i}(x)=\left[\begin{array}{c}
k_{1}-k_{2} x_{1}-k_{3} x_{1} x_{3} \\
k_{3} x_{1} x_{3}-k_{4} x_{2} \\
k_{5} x_{2}-k_{6} x_{3}
\end{array}\right]+u_{i} f_{i}(x)
$$

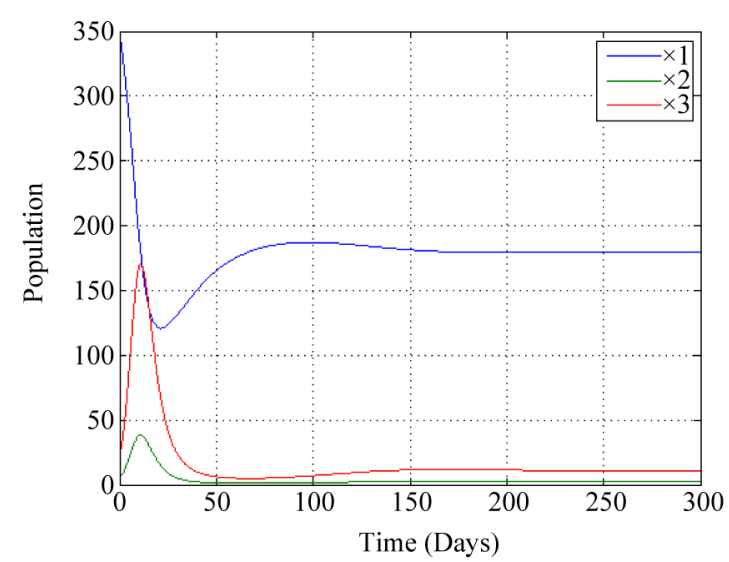

Figure 1. HIV-1 model without CLF, $x_{1}(0)=350, x_{2}(0)=5$, $x_{3}(0)=25$. \#

Table 1. Parameter values used in HIV-1 infection model.

\begin{tabular}{ccc}
\hline Parameter & Value & Unit \\
\hline$k_{1}$ & 10 & cells mm $^{3^{-1}}$ day $^{-1}$ \\
$k_{2}$ & 0.05 & day $^{-1}$ \\
$k_{3}$ & $5 \times 10^{-4}$ & $\mathrm{~mm}^{3}$ cells $^{-1} \cdot$ day $^{-1}$ \\
$k_{4}$ & 0.4 & day $^{-1}$ \\
$k_{5}$ & 40 & day $^{-1}$ \\
$k_{6}$ & 9 & day $^{-1}$ \\
\hline
\end{tabular}


The state feedback law with uncertainty is:

$$
u_{i}\left(x_{1}, x_{2}, x_{3}\right)=-\nabla V\left(x_{1}, x_{2}, x_{3}\right) f_{i}(x, \Delta)
$$

$V(x)$ from [21] is:

$$
\begin{aligned}
V(x, y, v)= & x^{*}\left(\frac{x}{x^{*}}-\ln \frac{x}{x^{*}}\right)+y^{*}\left(\frac{y}{y^{*}}-\ln \frac{y}{y^{*}}\right) \\
& +\frac{k_{4}}{k_{5}} v^{*}\left(\frac{v}{v^{*}}-\ln \frac{v}{v^{*}}\right)
\end{aligned}
$$

Now, we can express $V(x, y, v)$ as:

$$
\begin{aligned}
V\left(x_{1}, x_{2}, x_{3}\right)= & x_{1}^{*}\left(\frac{x_{1}}{x_{1}^{*}}-\ln \frac{x_{1}}{x_{1}^{*}}\right)+x_{2}^{*}\left(\frac{x_{2}}{x_{2}^{*}}-\ln \frac{x_{2}}{x_{2}^{*}}\right) \\
& +\frac{k_{4}}{k_{5}} x_{3}^{*}\left(\frac{x_{3}}{x_{3}^{*}}-\ln \frac{x_{3}}{x_{3}^{*}}\right)
\end{aligned}
$$

From $V\left(x_{1}, x_{2}, x_{3}\right)$ we can find $\nabla V\left(x_{1}, x_{2}, x_{3}\right)$ :

$\nabla V\left(x_{1}, x_{2}, x_{3}\right)=\left[\left(1-\frac{x_{1}^{*}}{x_{1}}\right)\left(1-\frac{x_{2}^{*}}{x_{2}}\right) \frac{k_{4}}{k_{5}}\left(1-\frac{x_{3}^{*}}{x_{3}}\right)\right]$

where $R_{0}=\frac{k_{1} k_{3} k_{5}}{k_{2} k_{4} k_{6}}, \quad x_{1}^{*}=\frac{k_{1}}{k_{2} R_{0}}$,

$$
x_{2}^{*}=\frac{k_{2} k_{6}}{k_{3} k_{5}}\left(R_{0}-1\right), \quad x_{3}^{*}=\frac{k_{2}}{k_{3}}\left(R_{0}-1\right)
$$

From Table 1 we can find $R_{0}$ and the equilibrium states:

$$
R_{0}=\frac{10}{9}, x_{1}^{*}=180, x_{2}^{*}=2.5, \quad x_{3}^{*}=\frac{100}{9}
$$

\subsection{Applying Control Strategy into Infected Cells with Uncertainty}

In this section, we can apply the control input to infected cells for HIV-1 infection model with uncertainty as:

$$
\begin{aligned}
\dot{x} & =f_{o}(x, \Delta)+\sum_{i=1}^{m} u_{i} f_{i}(x, \Delta) \\
& =\left[\begin{array}{c}
k_{1}-\left(k_{2} \pm \Delta\right) x_{1}-k_{3} x_{1} x_{3} \\
k_{3} x_{1} x_{3}-\left(k_{4} \pm \Delta\right) x_{2} \\
k_{5} x_{2}-\left(k_{6} \pm \Delta\right) x_{3}
\end{array}\right]+u_{1}\left[\begin{array}{c}
0 \\
\left(k_{6} \pm \Delta\right) x_{2} \\
0
\end{array}\right]
\end{aligned}
$$

In this case, $u_{1}\left(x_{1}, x_{2}, x_{3}\right)$ will be:

$$
\begin{aligned}
& u_{1}\left(x_{1}, x_{2}, x_{3}\right) \\
= & -\left[\left(1-\frac{x_{1}^{*}}{x_{1}}\right)\left(1-\frac{x_{2}^{*}}{x_{2}}\right) \frac{\left(k_{4} \pm \Delta\right)}{\left(k_{5} \pm \Delta\right)}\left(1-\frac{x_{3}^{*}}{x_{3}}\right)\right]\left[\begin{array}{c}
0 \\
\left(k_{6} \pm \Delta\right) x_{2} \\
0
\end{array}\right] \\
u_{1}\left(x_{1}, x_{2}, x_{3}\right) & =-\left(k_{6} \pm \Delta\right) x_{2}\left(1-\frac{x_{2}^{*}}{x_{2}}\right)
\end{aligned}
$$

\subsection{Applying Control Strategy into Virus with Uncertainty}

We can also apply the control input to virus for the HIV1 infection model with uncertainty as:

$$
\begin{aligned}
\dot{x} & =f_{o}(x, \Delta)+\sum_{i=1}^{m} u_{i} f_{i}(x, \Delta) \\
& =\left[\begin{array}{c}
k_{1}-\left(k_{2} \pm \Delta\right) x_{1}-k_{3} x_{1} x_{3} \\
k_{3} x_{1} x_{3}-\left(k_{4} \pm \Delta\right) x_{2} \\
k_{5} x_{2}-\left(k_{6} \pm \Delta\right) x_{3}
\end{array}\right]+u_{1}\left[\begin{array}{c}
0 \\
0 \\
x_{3}
\end{array}\right]
\end{aligned}
$$

In this case, $u_{1}\left(x_{1}, x_{2}, x_{3}\right)$ will be:

$$
\begin{gathered}
u_{1}\left(x_{1}, x_{2}, x_{3}\right) \\
=-\left[\left(1-\frac{x_{1}^{*}}{x_{1}}\right)\left(1-\frac{x_{2}^{*}}{x_{2}}\right) \frac{\left(k_{4} \pm \Delta\right)}{\left(k_{5} \pm \Delta\right)}\left(1-\frac{x_{3}^{*}}{x_{3}}\right)\right]\left[\begin{array}{c}
0 \\
0 \\
x_{3}
\end{array}\right] \\
u_{1}\left(x_{1}, x_{2}, x_{3}\right)=-x_{3} \frac{\left(k_{4} \pm \Delta\right)}{\left(k_{5} \pm \Delta\right)}\left(1-\frac{x_{3}^{*}}{x_{3}}\right)
\end{gathered}
$$

\section{Noise Effect on HIV-1 Dynamic System}

In this section, noise effect on HIV-1 dynamic system with external control input is investigated into two strategies as:

$$
\begin{aligned}
\dot{x}= & f_{o}(x)+\sum_{i=1}^{m} u_{i} f_{i}(x,)+d=\left[\begin{array}{c}
k_{1}-k_{2} x_{1}-k_{3} x_{1} x_{3} \\
k_{3} x_{1} x_{3}-k_{4} x_{2} \\
k_{5} x_{2}-k_{6} x_{3}
\end{array}\right] \\
& +u_{i} f_{i}(x)+d
\end{aligned}
$$

where $d$ represents noise effect.

\section{Simulation and Results}

In Figure 2, it is assumed a ( $\pm 5 \%)$ deterministic uncertainty in decay parameters of HIV-1 model are related to the first strategy. It's noted that uncertainty doesn't affect the control role on reducing viral load to an undetectable level, however the number of healthy cells with $(+5 \%)$ uncertainty is reduced and with (-5\%) uncertainty is increased and this can be referred to detrimental and beneficial perturbation respectively.

In Figure 3, it is assumed a high deterministic uncertainty $( \pm 20 \%)$ in decay parameters of HIV-1 model are related to the first strategy. It is noted that even for high uncertainty, the control is still effective on reducing viral load to an undetectable level, however the number of healthy cells with $(+20 \%)$ and $(-20 \%)$ uncertainty is reduced (detrimental perturbation) and increased (beneficial perturbation) respectively.

In Figure 4, it is assumed a ( $\pm 5 \%)$ deterministic uncertainty in decay parameters of HIV-1 model are related 

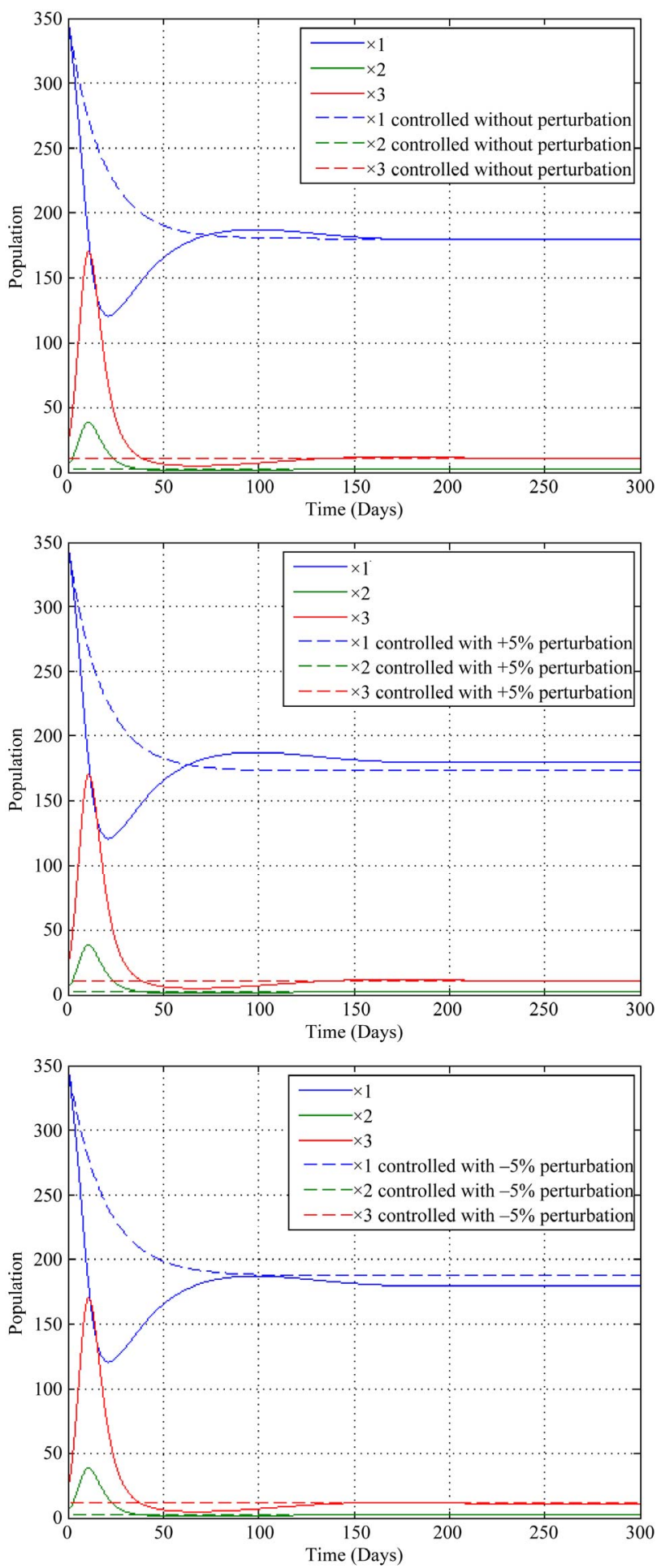

Figure 2. Control performance for nonlinear HIV-1 model under $\pm 5 \%$ deterministic parameters uncertainty for first strategy.

to the second strategy. It's noted that uncertainty affect the control role on reducing viral concentration to an undetectable level which means that the first strategy is more efficient than second strategy.
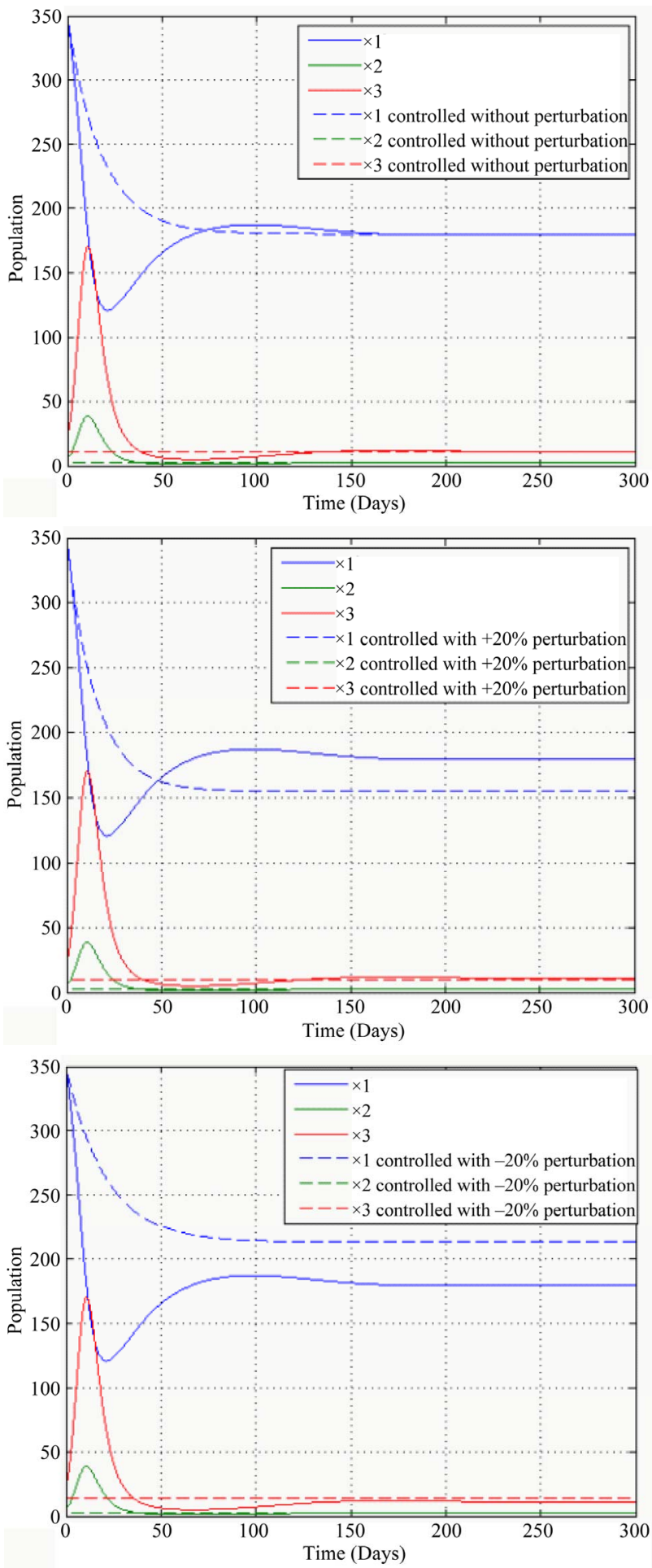

Figure 3. Control performance for nonlinear HIV-1 model under $\pm \mathbf{2 0} \%$ deterministic parameters uncertainty for first strategy.

In Figure 5, it is assumed a high ( $\pm 20 \%)$ deterministic uncertainty in decay parameters of HIV-1 model are related to the second strategy. It's noted at high uncertainty, the control effect becomes worse on reducing viral con- 

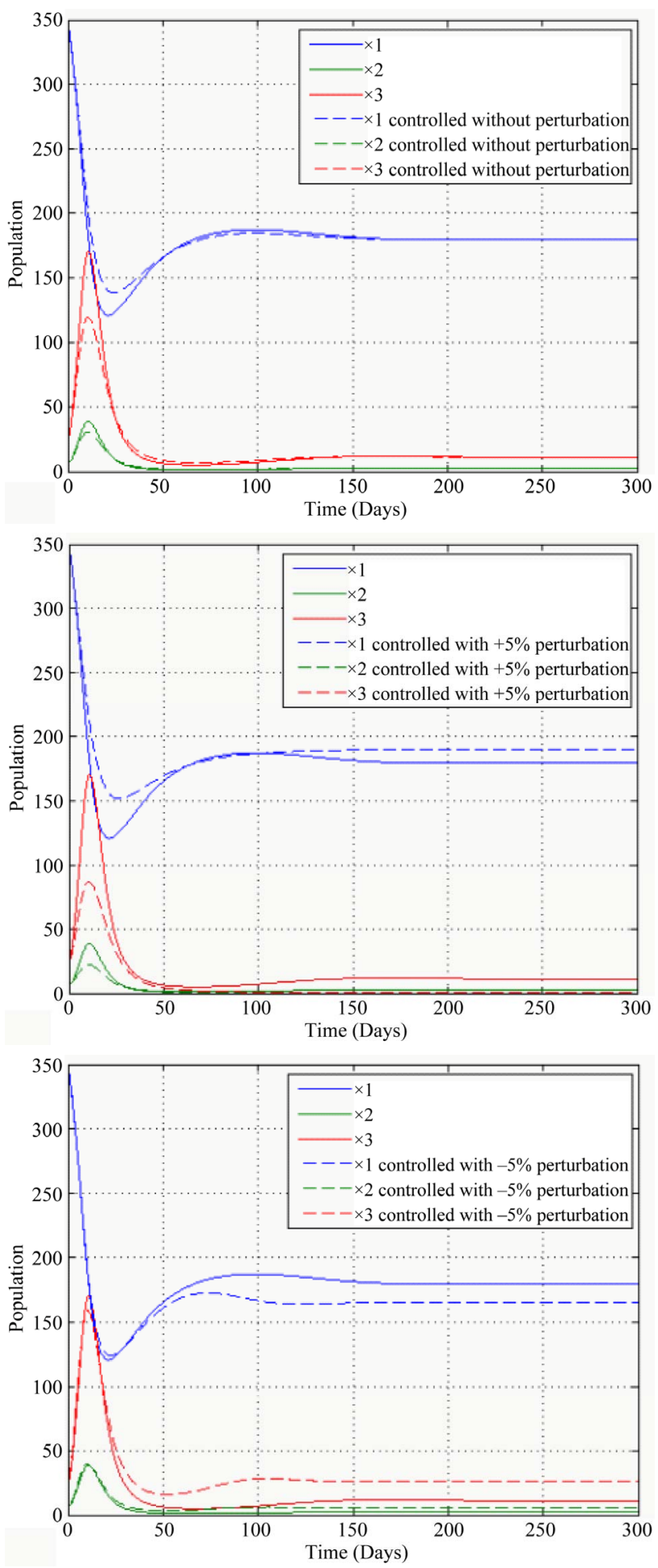

Figure 4. Control performance for nonlinear HIV-1 model under $\pm 5 \%$ deterministic parameters uncertainty for second strategy.

centration.

In Figure 6, we add a constant noise $(+10)$ in HIV-1 model related to first and second strategy. It's shown that noise has a little impact on the HIV-1 system dynamic
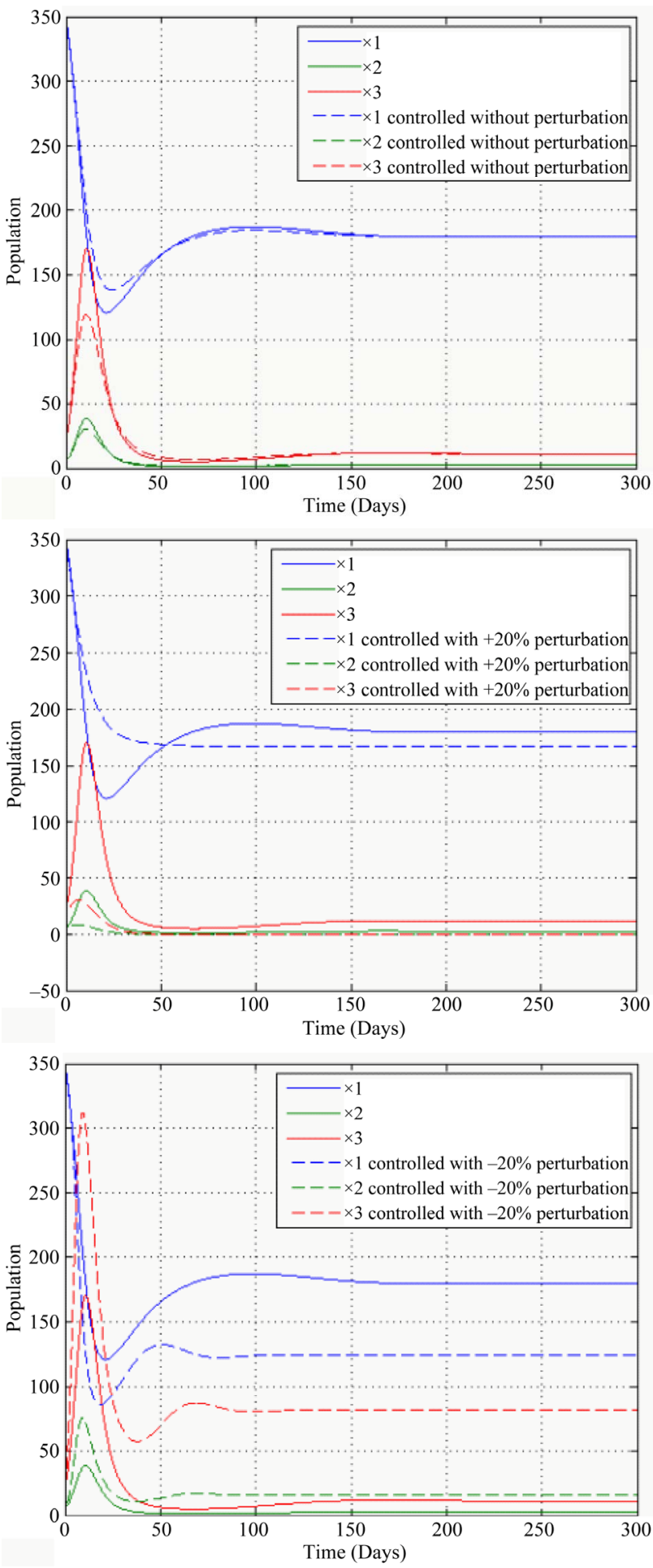

Figure 5. Control performance for nonlinear HIV-1 model under $\pm \mathbf{2 0} \%$ deterministic parameters uncertainty for second strategy.

and that is also depends on how much noise is added.

\section{Conclusion}

This paper has presented a new robust CLF control de- 

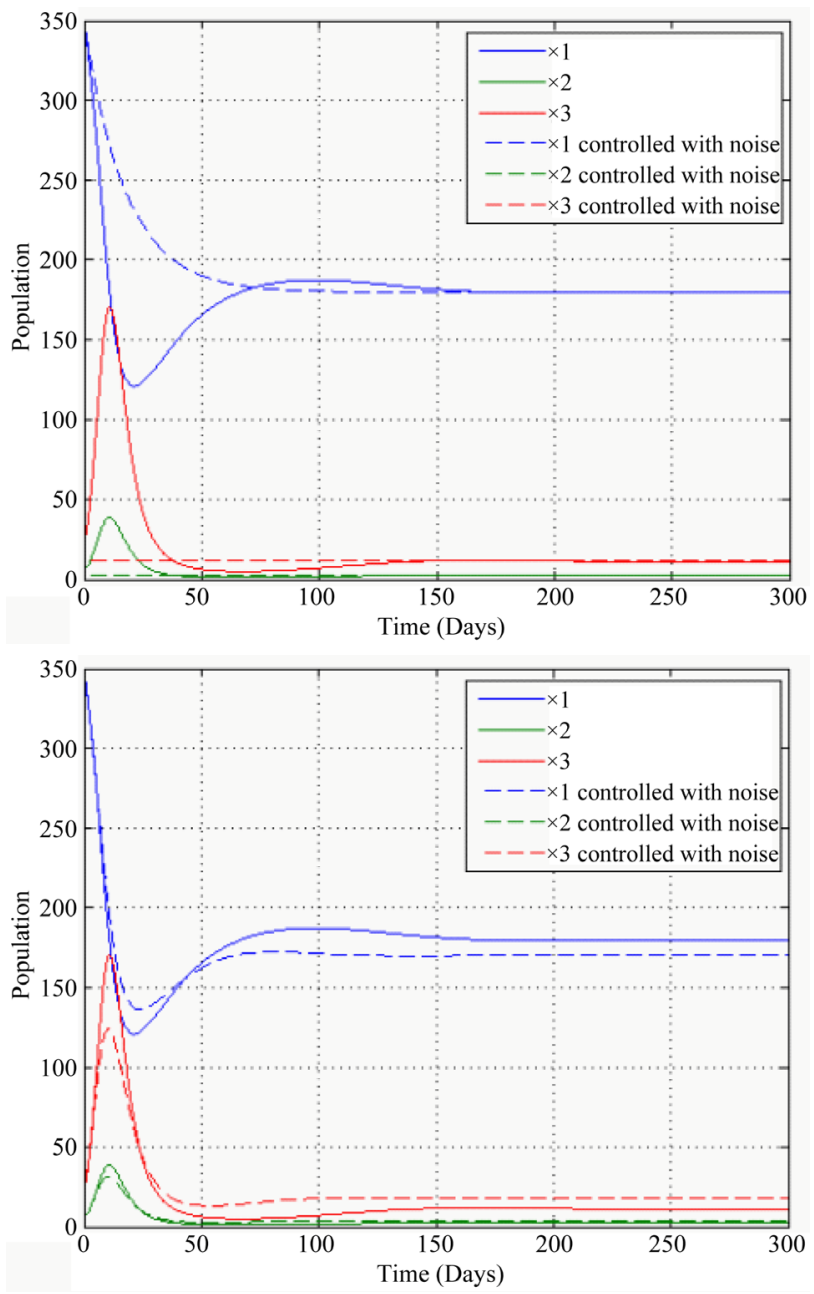

Figure 6. Noise effect on nonlinear HIV-1 model for first and second strategies respectively.

sign for uncertain and nonlinear HIV-1 infection models. We have found suitable nonlinear controller strategy to cause reduction of virus concentration to an undetectable level. Different strategies were considered to evaluate the control effectiveness. Additional noise into the HIV-1 system's dynamics was simulated to further test the nonlinear control strategies stochastic behavior.

\section{REFERENCES}

[1] D. M. Bortz and P. W. Nelson, "Sensitivity Analysis of a Nonlinear Lumped Parameter Model of HIV Infection Dynamics," Bulletin of Mathematical Biology, Vol. 66, No. 5, 2004, pp. 1009-1026. doi:10.1016/j.bulm.2003.10.011

[2] N. I. Stilianakis, K. Dietz and D. Schenzle "Analysis of a Model for the Pathogenesis of AIDS,” Mathematical Biosciences, Vol. 145, No. 1, 1997, pp. 27-46. doi:10.1016/S0025-5564(97)00018-7

[3] A. S. Perelson and P. W. Nelson, "Mathematical Analysis of HIV-1 Dynamics in Vivo," Society for Industrial and
Applied Mathematics Review, Vol. 41, No. 1, 1999, pp. 344. doi:10.1137/S0036144598335107

[4] M. A. Stafford, L. Corey, Y. Cao, E. S. Daar, D. D. Ho and A. S. Perelson, "Modeling Plasma Virus Concentration during Primary HIV Infection,” Journal of Theoretical Biology, Vol. 203, No. 3, 2000, pp. 285-301. doi:10.1006/jtbi.2000.1076

[5] A. S. Perelson, A. U. Neumann, M. Markowitz, M. Markowitz, J. M. Leonard and D. D. Ho, "HIV-1 Dynamics in Vivo: Virion Clearance Rate, Infected Cell Lifespan, and Viral Generation Time,” Science, Vol. 271, No. 5255, 1996, pp. 1582-1586. doi:10.1126/science.271.5255.1582

[6] V. Gajic, "Optimal Control of HIV-Virus Dynamics," Annals of Biomedical Engineering, Vol. 37, No. 6, 2009, pp. 1251-1261. doi:10.1007/s10439-009-9672-7

[7] S. Ge, Z. Tian and T. Lee, "Nonlinear Control of Dynamic Model of HIV-1,” IEEE Transaction on Biomedical Engineering, Vol. 52, No. 6, 2005, pp. 353-361. doi:10.1109/TBME.2004.840463

[8] M. Brandt and G. Chen, "Feedback Control of a Biodynamical Model of HIV-1,” IEEE Transaction on Biomedical Engineering, Vol. 48, No. 7, 2001, pp. 754-759. doi:10.1109/10.930900

[9] I. Craig and X. Xia, “Can HIV/AIDS Be Controlled,” IEEE Control Systems Magazine, Vol. 25, No. 1, 2005, pp. 8083. doi:10.1109/MCS.2005.1388805

[10] E. Sontag, "Some New Directions in Control Theory Inspired by Systems Biology,” Systems Biology, Vol. 1, No. 1, 2004, pp. 9-18. doi:10.1049/sb:20045006

[11] H. Chang and A. Astolfi, "Immune Response’s Enhancement via Controlled Drug Scheduling,” IEEE Conference on Decision and Control, New Orleans, 12-14 December 2007, pp. 3919-3924. doi:10.1109/CDC.2007.4434462

[12] L. Praly and Y. Wang, "Stabilization in Spite of Matched Un-Modeled Dynamics and an Equivalent Definition of Input-to-State Stability," Mathematics of Control, Signals and Systems, Vol. 9, No. 1, 1996, pp. 1-33. doi:10.1007/BF01211516

[13] R. Sepulchre, M. Jankovic and P. Kokotovic, "Constructive Nonlinear Control,” Springer-Verlag, Berlin, 1997.

[14] L. Faubourg and J. Pomet, "Design of Control Lyapnov Functions for 'Jurdjevic-Quinn' Systems,” Satbility and Stabilization of Nonlinear Systems, Vol. 246, 1999, pp. 137-150. doi:10.1007/1-84628-577-1_7

[15] A. Bacciotti, "Local Stabilizability of Nonlinear Control Systems,” World Scientific, Singapore, 1992.

[16] V. Jurjevic and J. Quinn, “Controllability and stability,” Journal of Differential Equations, Vol. 28, No. 3, 1978, pp. 381-389. doi:10.1016/0022-0396(78)90135-3

[17] R. Outbib and G. Sallet, "Stabilizability of the Angular Velocity of a Rigid Body Revisited," Systems and Control Letters, Vol. 18, No. 2, 1992, pp. 93-98. doi: 10.1016/0167-6911(92)90013-I

[18] A. Knorr and R. Srivastava, "Evaluation of HIV-1 Kinetic Models using Quantitative Discrimination Analysis,” Bioinformatics, Vol. 21, No. 8, 2004, pp. 1668-1677. doi:10.1093/bioinformatics/bti230 
[19] V. Yadav and S. N. Balakrishnan, “Optimal Impulse Control of Systems with Control Constraints and Application to HIV Treatment," Proceedings of the 2006 American Control Conference, Minneapolis, 14-16 June 2006, pp. 4824-4829. doi.org/10.1109/ACC.2006.1657484

[20] R. Zurakowski and A. R. Teel, "Enhancing Immune Response to HIV Infection Using MPC-Based Treatment
Scheduling," Proceedings of the 2003 American Control Conference, Denver, 4-6 June 2003, pp. 1182-1187. doi:10.1109/ACC.2003.1239748

[21] A. Korobeinikov, "Global Properties of Basic Virus Dynamics Models,” Bulletin of Mathematical Biology, Vol. 66, No. 4, 2004, pp. 879-883. doi:10.1016/j.bulm.2004.02.001 